\title{
OFDM FOR UNDERWATER ACOUSTIC COMMUNICATIONS: ADAPTIVE SYNCHRONIZATION AND SPARSE CHANNEL ESTIMATION
}

\author{
Milica Stojanovic \\ Massachusetts Institute of Technology \\ Cambridge, MA 02139
}

\begin{abstract}
A phase synchronization method, which provides non-uniform frequency offset compensation needed for wideband OFDM [1], is coupled with low-complexity channel estimation in the time domain. Sparsing of the channel impulse response leads to an improved performance, while adaptive synchronization supports decision-directed operation and yields low overhead. System performance is demonstrated using experimental data transmitted over a $1 \mathrm{~km}$ shallow water channel in the $19 \mathrm{kHz}$ $31 \mathrm{kHz}$ band.
\end{abstract}

Index Terms - Underwater acoustic communications, nonuniform Doppler distortion, channel sparsing, OFDM.

\section{INTRODUCTION}

The major problem in applying OFDM to underwater channels is the motion-induced Doppler distortion which creates non-uniform frequency offset in a wideband acoustic signal. Previous work on this problem has focused on two approaches: adaptive synchronization, which requires little overhead but relies on coherence between adjacent OFDM blocks [1], and non-adaptive synchronization, which requires null subcarriers to gain robustness to fast channel variations [2].

Here, we extend the approach [1] by coupling it with channel estimation in the time domain (impulse response). The motivation for doing so is the possibility to perform channel sparsing. Channel impulse response is often shorter than an OFDM block, and can thus be represented by fewer than $K$ coefficients that it takes to represent its transfer function on the $K$ subcarriers. A certain number $L<K$ coefficients of the time-domain response can be efficiently estimated using $L$ equally-spaced data symbols [3], a method used in [2]. We suggest a slight but important modification to this method to make it applicable to a general underwater channel, where the strongest signal arrival may not be the first one. Sparsing is implemented in an optimal manner simply by magnitude truncation of the time-domain channel coefficients. When the channel is truly sparse, performance improvement results from eliminating the unnecessary noise present in the full-size (overparametrized) channel estimate.

This work was supported by the ONR grants N00014-07-1-0202/0738.

\section{ALGORITHM OVERVIEW}

The received signal after FFT demodulation is modeled as

$$
\mathbf{y}_{k}(n)=\mathbf{H}_{k}(n) d_{k}(n) e^{j \theta_{k}(n)}+\mathbf{z}_{k}(n), k=0, \ldots K-1
$$

where $d_{k}(n)$ is the data symbol transmitted on the $k$ th subcarrier during the $n$-th OFDM block, $\mathbf{H}_{k}(n)$ is the channel transfer function evaluated at frequency $f_{k}=f_{0}+k \Delta f$ during the $n$-th block, and $\mathbf{z}_{k}(n)$ represents additive noise and any residual inter-carrier interference. Each boldface quantity contains $M$ entries corresponding to the spatially distributed receiver elements. The subcarrier spacing is $\Delta f=B / K$, the duration of one OFDM symbol is $T=1 / \Delta f$, and the duration of one block is $T^{\prime}=T+T_{g}$, where $T_{g}$ is the guard interval. The phase distortion $\theta_{k}(n)$ on an acoustic channel is modeled as

$$
\theta_{k}(n)=\theta_{k}(n-1)+a(n) \cdot 2 \pi f_{k} \cdot T^{\prime}
$$

where $a(n)$ is the Doppler rate, which is assumed to be constant during one OFDM block, but may change from one block to another. The physical interpretation of this parameter is that of the ratio of the relative transmitter/receiver velocity to the speed of sound $(1500 \mathrm{~m} / \mathrm{s}$ underwater). Non-uniform Doppler compensation [1] is based on estimating the parameter $a(n)$, and using this single estimate to compute the phases for all the subcarriers, thus keeping the computational complexity of phase synchronization at a minimum.

Time-domain channel estimation is based on defining the discrete Fourier pair

$$
\mathbf{H}_{k}(n)=\sum_{l=0}^{K-1} \mathbf{h}_{l}(n) e^{-j 2 \pi k l / K}
$$

Using this relationship, the frequency-domain estimates $\hat{\mathbf{H}}_{k}(n)$ needed for subsequent data detection are computed from the time-domain estimates $\hat{\mathbf{h}}_{l}(n)$. Adaptive channel estimation is accomplished using the following algorithm:

$$
\hat{\mathbf{h}}_{l}(n+1)=\lambda \hat{\mathbf{h}}_{l}(n)+(1-\lambda) \mathbf{x}_{l}(n)
$$

where

$$
\begin{array}{r}
\mathbf{x}_{l}(n)=\frac{1}{K} \sum_{k=0}^{K-1} \mathbf{X}_{k}(n) e^{+j 2 \pi k l / K}, \quad l=0, \ldots K-1 \\
\mathbf{X}_{k}(n)=\mathbf{y}_{k}(n) e^{-j \hat{\theta}_{k}(n)} \tilde{d}_{k}^{*}(n) \quad k=0, \ldots K-1
\end{array}
$$


The phase estimates $\hat{\theta}_{k}(n)$ and the symbol decisions $\tilde{d}_{k}(n)$ are obtained according to the algorithm [1].

If the channel is sparse, i.e. some of the coefficients $\mathbf{h}_{k}(n)$ are zero, the corresponding estimates can (and should) be discarded. By doing so, the problem dimensionality is reduced to the one dictated by the physics of propagation, and not by the number of subcarriers. Out of the $K$ coefficients, $J$ to be kept are selected as those whose magnitude is below some threshold (e.g., $15 \mathrm{~dB}$ below the maximum). If the input noise is white, so is the noise that affects the time-domain channel estimate, and, hence, sparsing is performed optimally by truncation in magnitude.

Time-domain estimation requires an FFT/IFFT to compute (3) and (5). When the total span (delay spread) of significant coefficients is limited, an IFFT of size $L<K$ can be used to compute (5). In order to do so, $L$ is selected as the first power-of-two integer greater than the delay spread, and the input to the IFFT is formed by taking every $K / L$-th subchannel of $\mathbf{X}_{k}(n)$. Specifically, we now have that

$$
\mathbf{x}_{l}(n)=\frac{1}{L} \sum_{k=0}^{L-1} \mathbf{X}_{k K / L}(n) e^{+j 2 \pi k l / L}, l=0, \ldots L-1
$$

which reduces to (5) when $L=K$. Channel updating (4) and sparsing are performed as before.

To reconstruct the frequency-domain values (3), an FFT of size $K$ must be applied. A word of caution is in order regarding this step. Normally, it is assumed that significant channel coefficients are those with indices $0 \div(L-1)$, and, hence, the frequency-domain response is computed by appending $K-L$ zeros to the estimates $\hat{\mathbf{h}}_{0}(n), \ldots \hat{\mathbf{h}}_{L-1}(n)$ before taking the FFT [3]. This approach befits a minimumphase (causal) channel, in which the strongest time-domain coefficient $\mathbf{h}_{0}(n)$ is followed by other coefficients. However, an underwater acoustic channel may not conform to this model, in which case its significant coefficients are those with indices $(-A) \div(L-1-A)$, or, equivalently from the viewpoint of the model (3), those with indices $0 \div(L-1-A)$ and $(K-A) \div(K-1)$, where $A$ denotes the length of the anti-causal part. The IFFT of size $L<K$ can still be applied to obtain the $L$ (contiguous) time-domain coefficients, but these coefficients have to be interpreted properly. Namely, the $K-L$ zeros should not be appended, but inserted before the last $A$ coefficients. The value of $A$ may not be known exactly, but it can be determined approximately from a prior channel measurement, or general channel statistics. The place where the zeros should be inserted can also be determined online, by observing the initial channel estimate. (It will be easier to do this for a greater $L$, and also when the response is truncated).

If the channel span is indeed limited to within $L$ coefficients, in the presence of white noise, the error variance of the re-computed frequency-domain coefficients is the same as if they were estimated directly [3]. While direct estimation in the frequency domain requires all $K$ data symbols, indirect

\begin{tabular}{|l||r|r|r|r|}
\hline$B=12 \mathrm{kHz}$ & $K$ & $\Delta f[\mathrm{~Hz}]$ & $T[\mathrm{~ms}]$ & $B T_{g} / K$ \\
\hline$f_{0}=19 \mathrm{kHz}$ & 128 & 93.75 & 10.6 & 2.34 \\
\hline$f_{s}=96 \mathrm{kHz}$ & 256 & 46.87 & 21.3 & 1.17 \\
\hline$T_{g}=25 \mathrm{~ms}$ & 512 & 23.40 & 42.6 & 0.58 \\
\hline$N_{d}=2^{15}$ & 1024 & 11.71 & 85.3 & 0.29 \\
\hline QPSK & 2048 & 5.86 & 170.7 & 0.14 \\
\hline
\end{tabular}

Table 1. OFDM signal parameters used for the experiment.

estimation in the time-domain requires only $L$. If the channel is truly sparse, and only the $J$ significant coefficients out of $L$ are kept, the error variance will be reduced by a factor of $L / J$ [4].

The $L$ data symbols needed to estimate the channel in the time-domain must be the a-priori known pilots if non-adaptive processing is used. With adaptive processing, symbol decisions are also available, which can be made for a current block using channel estimates from the previous one. Some of the pilots (or all, after initial training) can thus be eliminated to reduce the overhead. Moreover, channel estimation in the time domain can be performed using all $K$ symbol decisions instead of $L$ only. This approach will require the fullsize IFFT (5), but the error variance will improve by a factor of $K / L$ if the decisions are correct. Note that sparsing helps to reduce error propagation, as fewer symbol decisions contribute to the channel estimates. Together with sparsing, the overall improvement in the error variance of the re-computed frequency-domain coefficients will be $K / J$, as compared to their direct estimation.

While the number of significant impulse response coefficients $J$ is dictated mostly by the physics of propagation, the number of subcarriers $K$ is a system design parameter. By increasing $K$, the bandwidth efficiency increases, and, hence, it is of interest to make $K$ as large as possible. The maximal value of $K$ that can be used in a low-complexity OFDM system based on post-FFT processing is that for which coherence over one symbol interval $T=K / B$ can still be maintained. In a wideband acoustic system, such as the one we will discuss in the following section, $K$ can be as large as 1024. For such values of $K$, and a modest number of distinct propagation paths, one can expect non-negligible improvements from sparse time-domain channel estimation.

\section{EXPERIMENTAL RESULTS}

The experimental data were collected by the Woods Hole Oceanographic Institution (WHOI) team during the "AUV Fest" held at the Panama City Beach, FL, in June 2007. The transmitter and receiver were deployed $10 \mathrm{~m}$ below the surface in $20 \mathrm{~m}$ deep water, and separated by $1 \mathrm{~km}$. The receiver had an 8 element vertical array, spanning $2 \mathrm{~m}$. Table 1 summarizes the signal parameters. 
The factor $B T_{g} / K=\alpha$ determines the bandwidth efficiency, $R / B=1 /(1+\alpha)$ symbols/s/Hz. The signals were also coded, using the BCH $(64,10)$ code. This code was selected so as to match the one currently implemented in the WHOI modem. For $K$-carrier OFDM, each string of 10 input bits is encoded into a 32-symbol QPSK codeword, and $K / 32$ such codewords are collected. The so-obtained $K$ symbols are assigned to the OFDM subcarriers so that the symbols of one codeword are maximally separated in frequency (the first codeword modulates subcarriers $0,32,64$, etc.; the second 1 , 33,65 , etc., and so on). Soft decision decoding is employed.

Fig. 1 shows the received signal of a frame that we shall use for a case study. The substantial variation in the signal level that occurs over the frame duration is to be expected in a shallow water channel.

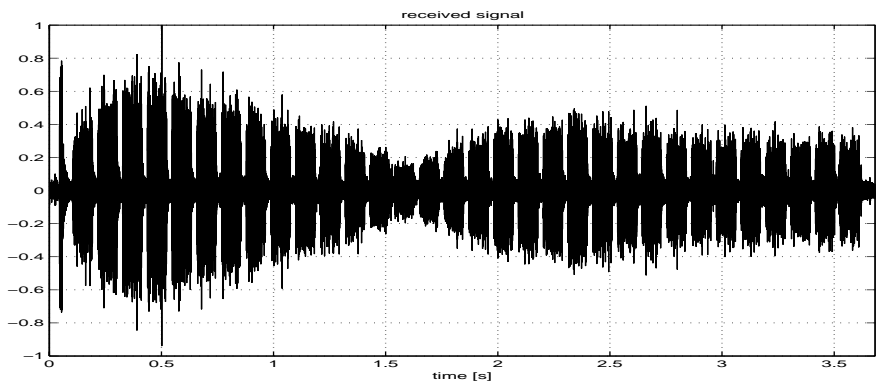

Fig. 1. Received signal of a $K=1024$ frame contains a preamble and $N_{d} / K=32$ OFDM blocks with zero padding.

Fig.2 shows the channel measurments obtained from the preamble. There is a fair degree of spatial variation between the top and the bottom element, and a moderate delay spread ( $L=32$ will suffice for $2.5 \mathrm{~ms}$ ). The closely spaced main arrivals may fluctuate in time/space, leading to a non-minimumphase channel.
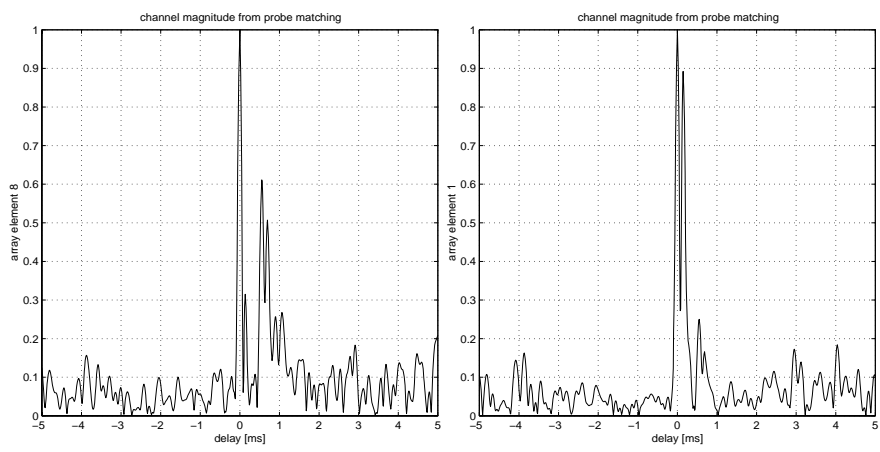

Fig. 2. Acquisition of the frame preamble at the top (left) and bottom elements of the array.

Fig. 3 shows the performance of a full-size receiver, which we use as a benchmark. The channel estimates clearly indicate overparametrization, i.e. the possibility to discard many of the $K$ coefficients used to represent the channel response.
When sparsing is implemented with the same receiver configuration (truncation threshold is set to 0.2 relative to the maximal coefficient amplitude), a $3 \mathrm{~dB}$ improvement is obtained. Fig.4 shows the number of channel coefficients (average over the receiver elements) kept for each OFDM block.

Identical performance is obtained using reduced-size $L=$ 128 IFFT and $K / L$ pilots, with anti-causal length $A=5$. At $L=32,1 \mathrm{~dB}$ is lost, and another at $L=16$.

In Fig.5, we address the performance in decision-directed mode. The receiver uses training only in the first OFDM block, while the remaining 31 blocks are detected without pilot assistance. The MSE penalty incurred is about $0.5 \mathrm{~dB}$. It is interesting to note the correlation between the signal level (Fig.1) and the symbol error rate pattern (plot labeled 'SERtime'). All of the results presented were generated without the decoder in the loop (high-frequency subbands are the obvious culprit for the high SER). When the channel code is exploited, there are no errors, and, hence, no penalty. Coding across frequency only, albeit suboptimal, allows instantaneous decoding of a block, i.e. higher reliability of symbol decisions used in adaptation.

In summary, suffice it to say that similar results were obtained with all $K<1024$, and, surprizingly, good performance was observed even with $K=2048$, although not consistently.

\section{CONCLUSIONS}

The results presented demonstrate the benefits of coupling adaptive phase synchronization [1] with time-domain channel estimation: low overhead is achieved by eliminating the need for null subcarriers; model-based phase prediction enables decision-directed operation which in turn reduces (or eliminates) the need for pilot subcarriers, and channel sparsing yields improved performance at complexity that does not have to grow as the number of subcarriers is increased for higher bandwidth efficiency.

\section{REFERENCES}

[1] M.Stojanovic, "Low complexity OFDM detector for underwater acoustic channels," IEEE Oceans Conf., Sept. 2006.

[2] B.Li, S.Zhou, M.Stojanovic, L.Freitag and P.Willet, "Non-uniform Doppler compensation for zeropadded OFDM over fast-varying underwater acoustic channels," IEEE Oceans Conf., June 2007.

[3] R.Negi and J.Cioffi, "Pilot tone selection for channel estimation in a mobile OFDM system," IEEE Trans. Consumer Electronics, vol.44, No.3, Aug. 1998.

[4] H.Minn and V.Bhargava, "An investigation into timedomain approach for OFDM channel estimation," IEEE Trans. Broadcasting, vol.46, No.4., Dec. 2000, pp. 240-248. 


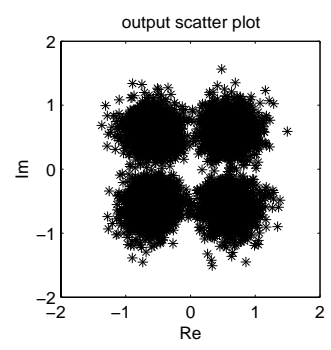

Performance:

MSE: $-7.9 \mathrm{~dB}$

SER: 0.0439 , uncoded

BER: 0, coded BCH $(64,10)$
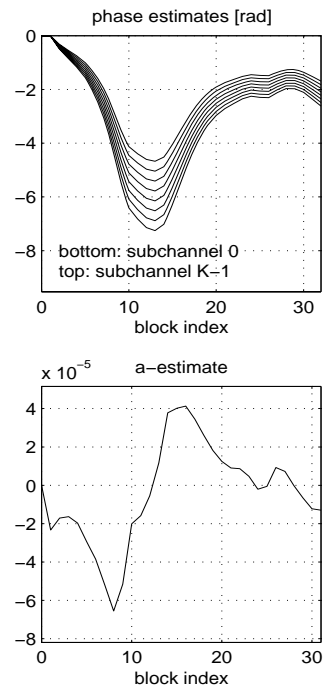

Fig. 3. Signal processing results: $K$-coefficient channel response estimation without sparsing.

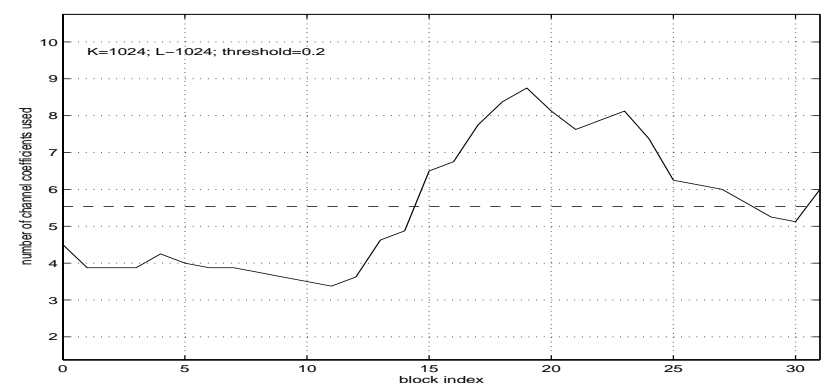

Fig. 4. Sparsing of the $K$-coefficient channel response estimate.
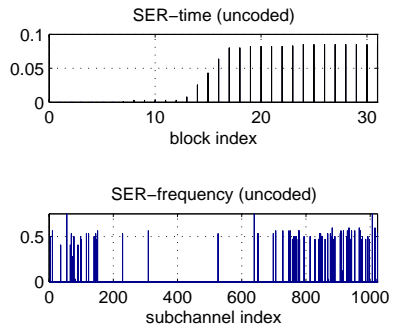
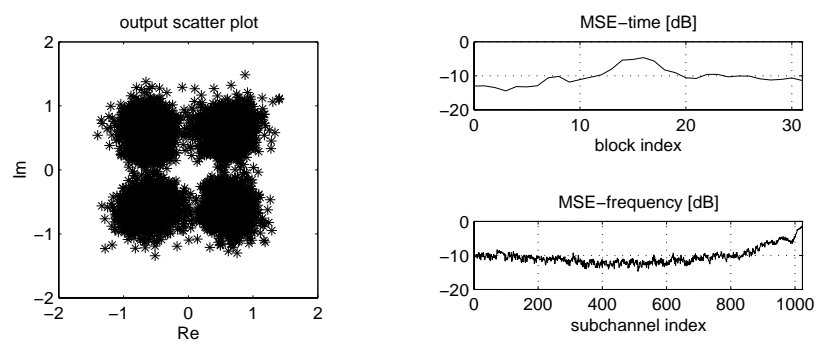

Performance:

MSE: $-9.5 \mathrm{~dB}$

SER: 0.0254 , uncoded

BER: 0, coded BCH $(64,10)$
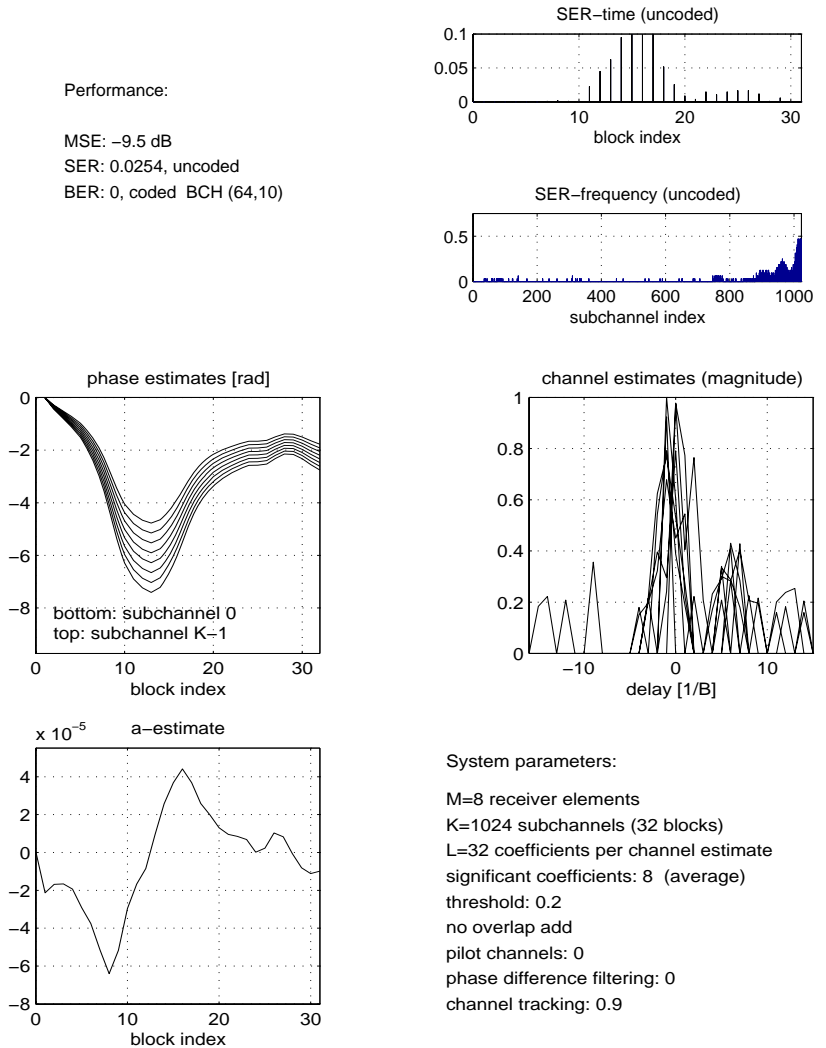

System parameters:

$\mathrm{M}=8$ receiver elements

$\mathrm{K}=1024$ subchannels ( 32 blocks)

$\mathrm{L}=32$ coefficients per channel estimate

significant coefficients: 8 (average)

threshold: 0.2

no overlap add

pilot channels: 0

phase difference filtering: 0

channel tracking: 0.9

Fig. 5. Signal processing results: $L$-coefficient channel response estimate with sparsing, no pilots.

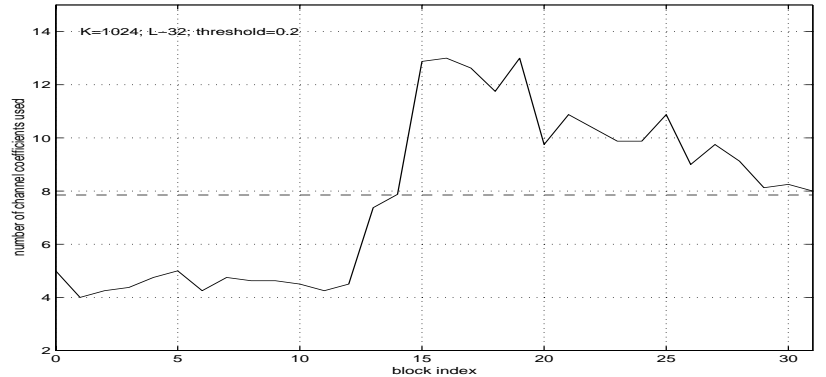

Fig. 6. Sparsing of the $L$-coefficient channel response estimate. (Sparsing patterns with and without pilots are similar.) 